\title{
Treatment of Ruptured Infected Abdominal Aortic Aneurysm Caused by Salmonella spp.: A Case Report
}

Katarzyna Napiórkowska-Baran (D) - Tomasz Rosada · Ewa Alska •

Adam Wawrzeńczyk · Zbigniew Bartuzi

Received: April 2, 2021 / Accepted: June 29, 2021 / Published online: July 12, 2021

(C) The Author(s) 2021

\section{ABSTRACT}

Despite significant advances in diagnostic methods, sepsis is still a current medical problem, and its complications contribute to increased mortality among different age groups. The article presents the case of a 71-year-old man who developed an inflammatory aneurysm of the abdominal aorta in the course of sepsis of Salmonella spp. etiology, followed by its rupture. The case confirms that we must not forget about rare, especially unusual complications, which may depend on the type of microorganism. Properly quick diagnosis allowed for the implementation of appropriate treatment and survival of the patient.

Keywords: Abdominal aortic aneurysm; Bleeding; Infected aneurysm; Salmonella spp; Sepsis

K. Napiórkowska-Baran ( $)$ - T. Rosada · E. Alska · A. Wawrzeńczyk · Z. Bartuzi Department and Clinic of Allergology, Clinical Immunology and Internal Diseases, Ludwik Rydygier Collegium Medicum in Bydgoszcz Nicolaus Copernicus University in Torun, University Hospital No. 2 dr J. Biziel in Bydgoszcz Ul. Ujejskiego 75, 85-164 Bydgoszcz, Poland e-mail: knapiorkowska@cm.umk.pl

\section{Key Summary Points}

Despite significant advances in diagnostic and therapeutic methods, sepsis is still a current medical problem

Salmonella spp. are the most common etiological agent of bacterial diarrhea around the world

Salmonella spp. may be an etiological factor of other, even serious diseases, such as infection of internal organs or joints and sepsis

Sepsis caused by Salmonella spp. increases the risk of aneurysm and its rupture

Salmonella spp. are responsible for more than $50 \%$ of infected aneurysms

Properly prompt diagnosis allows for the implementation of appropriate treatment and the patient's survival, despite the occurrence of complications of sepsis that directly threaten life 


\section{INTRODUCTION}

Despite significant advances in diagnostic methods, sepsis is still a current medical problem, and its complications contribute to increased mortality among different age groups. The incidence of sepsis worldwide is up to 535 cases per 100,000 person-years [1, 2]. Sepsis complications include meningitis, osteomyelitis, and endovascular changes, e.g., an aneurysm, as in the case described below [3].

Salmonella spp. are non-spore-forming, nonenveloped, gram-negative rods belonging to the Enterobacteriacae family, growing under aerobic conditions, but also capable of optional growth under anaerobic conditions. These bacteria cause salmonellosis, a widespread disease that may have two different courses, depending on whether the infection is caused by a typhoidal or non-typhoidal serovar $[4,5]$. Swallowed pathogens survive the low $\mathrm{pH}$ of gastric acid and also avoid numerous gastrointestinal defence mechanisms, gaining access to the intestinal epithelium. Salmonella rods preferentially integrate with $\mathrm{M}$ cells, which then transport the described microorganisms to the lymphoid cells (B and T) of Peyer's patches. After overcoming the epithelial barrier, serotypes associated with triggering a systemic disease penetrate into intestinal macrophages and spread in the reticuloendothelial system, which can lead, among others, to the formation of an infected aortic aneurysm, while non-typhoidal serotypes induce an early inflammatory response, which results, among others, in diarrhoea [4]. Non-typhoidal Salmonella (NTS) is a common cause of bacterial gastroenteritis worldwide, with 153 million cases leading to 57,000 deaths reported annually. Enteric fever caused by typhoidal Salmonella (TS) is a severe illness with significant rates of morbidity and mortality in many parts of the developing world, with an estimated 31 million cases leading to $>215,000$ deaths worldwide annually [6].

The risk factors for infection include: consumption of raw food ingredients that may have come into contact with infected animals or humans, improper storage or incomplete cooking of food products, living in regions with high sanitary and epidemiological risk, and immunodeficiency (both primary and secondary) [6]. The most important conservation factor is the implementation of strict hygiene practices. In a genome-wide association study of patients in Vietnam and Nepal with blood culture-confirmed enteric fever, a strong association was found for HLA-DRB1 as a major contributor to resistance against enteric fever, presumably through antigen presentation [7]. Research on Toll-like receptors (TLRs) and the cystic fibrosis transmembrane conductance regulator (CFTR) requires further research [6].

Population screening studies have determined that the prevalence of abdominal aortic aneurysms in individuals $>55$ years old is $4-7 \%$ in males and $1-2 \%$ in females. Aneurysm diameter remains the most frequently studied factor that is associated with the progression of aneurysm and determines the indication for treatment [8].

Mycotic aneurysm (MA) is a relatively rare but life-threatening disease, accounting for $0.06-2.6 \%$ of all aneurysms [9]. The English term-mycotic aneurysm-introduced by William Osler in 1885 to describe a case of the aortic arch rupture in the course of mycosis-like vegetations on the aortic valve, is quite misleading, because now the term is used in a broader sense and assigned to any inflammatory aneurysm or so-called infectious aortitis, regardless of pathogenesis $[9,10]$. According to Hartman et al., by 1965, Salmonella spp. were responsible for most cases of infected aortic aneurysms. Currently, the main aetiological factor of infected aortic aneurysm is Staphylococcus aureus, while Salmonella ranks second, accounting for about $10 \%$ of cases [11]. However, infected aortic aneurysm still remains the most common manifestation of a systemic Salmonella infection.

The aim of the study is to emphasise that life-threatening complications of infection may depend on the type of microorganism. Properly fast diagnosis allows for the implementation of appropriate treatment and the patient's survival. It is of particular importance in the era of constantly increasing frequency of infections. 


\section{CASE REPORT}

\section{Main Ailments and Medical History}

A 71-year-old man was admitted to hospital due to general body weakness and fever up to $42^{\circ} \mathrm{C}$ lasting about 3 days. Initially, the patient called the Emergency Ambulance Service and was given paracetamol. However, the symptoms recurred, which forced the patient to seek further help. All mentioned symptoms occurred for the first time in the patient's life. After reporting to the Hospital Emergency Department, laboratory and imaging tests were performed (discussed below), and a decision was made to hospitalise the patient in the department of internal medicine for further diagnosis and treatment.

In addition, this man had been treated with insulin for type 2 diabetes for several years and was taking drugs for hypertension irregularly. Apart from that, 12 years earlier, the patient had required surgical intervention because of a perforation of a duodenal ulcer in the course of an untreated peptic ulcer disease. Nicotine addiction was also found (approximately 54 packyears). The patient denied other chronic diseases, surgical interventions or hospitalisations.

\section{Physical Examination on Admission to Hospital}

The presence of abdominal scars after laparotomy was noticed, accompanied by a small abdominal hernia. Apart from that, numerous trophic changes and varicose veins were observed on the skin of both lower legs. Above the lung fields, there was normal vesicular sound with single basal rales. BP was $100 / 50 \mathrm{mmHg}, \mathrm{HR} 72 / \mathrm{min}$, respiratory rate 18/min, SpO2 95\% and Glasgow Coma Scale 15 points.

\section{Additional Tests on Admission to Hospital}

The following abnormalities in laboratory tests were found: WBC $13.85 \mathrm{G} / \mathrm{l}$ (N: 3.6-10.5 G / l), PT 17.2 s. (N: 9.4-13.9 sek.), creatinine $1.39 \mathrm{mg} /$ dl (N: $0.67-1.17 \mathrm{mg} / \mathrm{dl})$, glucose $206 \mathrm{mg} / \mathrm{dl}$,
C-reactive protein $122.3 \mathrm{mg} / \mathrm{l}(\mathrm{N}<5 \mathrm{mg} / \mathrm{l})$ and procalcitonin $1.76 \mathrm{ng} / \mathrm{ml}(\mathrm{N}<0.5 \mathrm{ng} / \mathrm{ml})$. The chest $\mathrm{x}$-ray showed blunting of the right costophrenic angle and streaky fibrous changes in the left costophrenic angle. An ultrasound of the abdominal cavity was performed, which showed: a gallbladder contracted on the deposits, a cyst in the left kidney $(8 \mathrm{~mm}$ in diameter) and a heterogeneous prostate enlarged to $80 \mathrm{ml}$. The abdominal aorta was assessed, and no evidence of the aneurysm was visible (examination performed by a specialist in radiology). Blood was also taken for microbiological testing.

\section{Course of hospitalisation}

The patient was treated with empiric ceftriaxone therapy ( $4 \mathrm{~g}$ daily). Due to the high risk of venous thromboembolic disease, evaluated on the basis of the Padua Prediction Score with the result of 5 points, anticoagulation prophylaxis was started in the form of $0.3 \mathrm{ml}$ low-molecularweight heparin per day.

On the 4th day of hospitalisation, the bacterium Salmonella spp. was isolated in two separate blood samples, sensitive among others for ticarcillin, piperacillin with tazobactam and ciprofloxacin.

Based on the performed examinations, the patient was diagnosed with Salmonella spp. sepsis, and after the microbiological consultation, ciprofloxacin at a dose of $800 \mathrm{mg}$ per day was added to empirically administered intravenous antibiotic therapy with cefritaxone, resulting in a slight improvement in the clinical condition and a slight reduction of inflammatory markers.

On the 5th day of hospitalisation late in the evening, the patient reported sudden weakness. Then, the patient went to the toilet with a loose stool, and after returning he complained of an unusually severe abdominal pain with the greatest intensity located within the left lower abdomen. The patient also had a few seconds of disorientation and thought blocking followed by full auto- and allopsychic orientation. The patient was given infusion fluids. Blood pressure was $160 / 80 \mathrm{mmHg}$, heart rate $100 / \mathrm{min}$, 
respiratory rate $22 / \mathrm{min}, \mathrm{SpO}_{2} 94 \%$ and Glasgow Coma Scale 11 points. After several dozen minutes, while standing up, the patient lost consciousness, slumping to the floor. In control laboratory tests, anaemia was found with a decrease in haemoglobin to $7.8 \mathrm{~g} / \mathrm{dl}(\mathrm{N}$ : $14-18 \mathrm{~g} / \mathrm{dl})$. The man was transferred to an intensive medical supervision room, where transfusion of 2 units of red blood cell concentrate, urgent surgical consultation and further diagnostics were recommended. Computed tomography of the abdominal cavity and pelvis showed the presence of aortic aneurysm, surrounded by a cuff of tissues, with an irregular flow channel, with dimensions of $35 \times 53 \mathrm{~mm}$, with active bleeding from the left lateral side (Diagnosis: ruptured infected aortic aneurysm) (Figs. 1 and 2). In addition, lymph nodes were enlarged to $18 \mathrm{~mm}$ at the level of the aneurysm; a large heterogeneous haematoma descending to the pelvis, with dimensions of $10 \times 11 \times 23$ $\mathrm{cm}$, was found in the left retroperitoneal space, with elevation and rotation of the left kidney with impaired perfusion as well as fluid in the pleural cavities and alectasis in the lower lung fields. After a telephone consultation with a vascular surgeon, the patient was transferred to

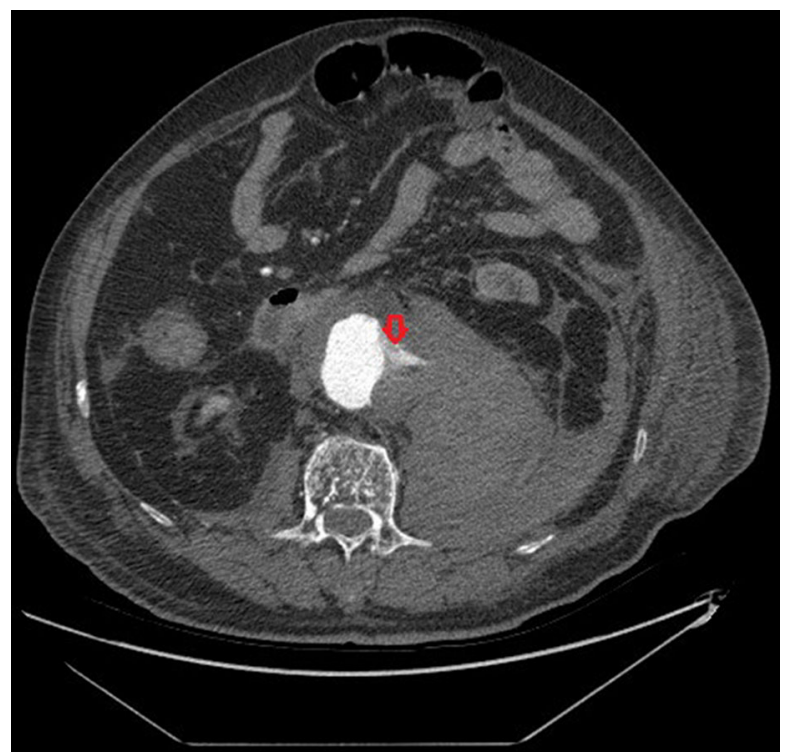

Fig. 1 Image of a ruptured inflammatory aortic aneurysm (CT) - extravasation of an intravenous contrast agent

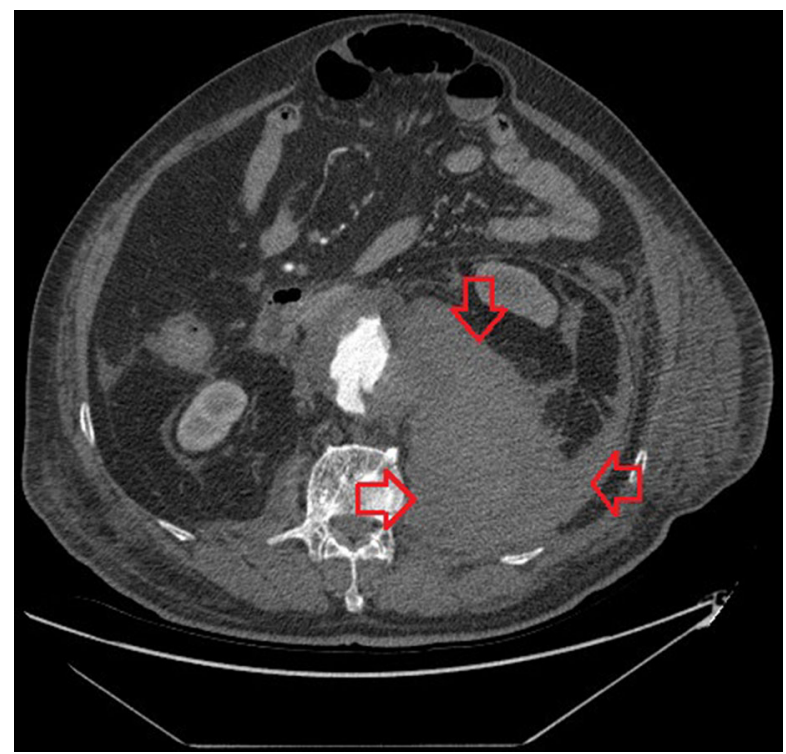

Fig. 2 Image of a ruptured inflammatory aortic aneurysm (CT) - haemorrhage into posterior pararenal and perirenal compartment

the vascular surgery department for urgent surgical treatment. The time from the onset of symptoms to the development of an aneurysm complicated by rupture was 8 days.

The patient, transferred in a state of haemorrhagic shock, underwent the emergency implantation of E-tegra stent graft in the abdominal aorta and common iliac arteries, thus excluding the aneurysm from the bloodstream. Improved clinical condition was observed during further hospitalisation. During the patient's stay at the Vascular Surgery Clinic, the patient underwent a number of laboratory and imaging tests and was consulted by an internist, who also diagnosed pneumonia with pleural effusion requiring thoracocentesis. After achieving clinical stabilisation of the patient, he was transferred to his previous department of internal medicine, where he underwent further conservative treatment and was observed for the next 24 days. After the resolution of the reported complaints and after achieving stabilisation of all vital signs, the patient was discharged home in good condition.

The man was referred to the Vascular Surgery Outpatient Clinic for further treatment and a 
controlled CT scan of the abdomen and pelvis was ordered, in accordance with the decision of the vascular surgeon. In addition, oral antibiotic therapy with $0.5 \mathrm{~g}$ ciprofloxacin per day in two divided doses was prescribed, at least until the follow-up appointment, according to the hospital microbiologist's recommendations.

\section{DISCUSSION}

The abdominal ultrasound examination is characterised by $95 \%$ sensitivity and nearly $100 \%$ specificity in the diagnosis of this disease [12]. The lack of visualisation of the aneurysm in the ultrasound examination of the described patient (performed on admission to hospital) confirms that the aneurysm arose in the course of the infection.

In order for MA to develop, disruption of the function or integrity of the vascular endothelium is necessary, which, for example, is the case with atherosclerosis. It is also suggested that traumatic injury of arteries, immunocompromised status, sepsis, endocarditis, congenital cardiovascular defects and connective tissue disorders also predispose to MA [10]. Other risk factors include smoking, hypertension, diabetes, etc. Initial symptoms are non-specific. High fever, chills and stomachache are typical [10]. There are no pathognomonic symptoms. If an aneurysm ruptures, the patient's condition deteriorates rapidly. Rapid diagnosis and surgical treatment are necessary. However, in the case of bacteraemia, it is necessary to institute empirical antibiotic therapy as soon as possible, which must be modified immediately after the antibiogram result is obtained.

There are no specific guidelines for empirical therapy in the case of suspected sepsis of Salmonella aetiology. It is possible to use intravenous ampicillin therapy at a dose of $100-200 \mathrm{mg} / \mathrm{kg} /$ day in four divided doses or a trimethoprim preparation with sulfometaxazole at a dose of $8-10 \mathrm{mg} / \mathrm{kg} /$ day in three divided doses. In the case of bacterial resistance to the above drugs, another option is third-generation cephalosporins such as cefotaxime or cefritaxone, but their intracellular concentration may not be sufficient. Currently, intravenous therapy with ciprofloxacin at a dose of $7.5 \mathrm{mg} /$ $\mathrm{kg}$ twice a day remains the gold standard, as it is a highly effective antibiotic, and it is also possible to easily replace intravenous therapy with oral therapy, which is very beneficial because of the necessity of long-term continuation of antibiotic therapy $[13,14]$.

\section{CONCLUSIONS}

Absence of an abdominal aortic aneurysm on admission to hospital, positive blood cultures (in the absence of other potential sources of infection), epidemiological data on MA and the absence of other factors that could induce an aneurysm confirm the causal relationship between infection with Salmonella spp. and the development of an aneurysm and its subsequent rupture.

Sepsis is a rapid, systemic inflammatory response of the body to an infection caused by bacteria, fungi or, much more rarely, viruses. Most often, it concerns elderly people with numerous other diseases; however, the occurrence among young people and children, especially newborns, is not uncommon. Clinical symptoms are usually non-specific, which usually hampers and delays the proper diagnosis.

Aortic aneurysm in the course of sepsis is not one of the most common complications, but its occurrence significantly worsens the patient's general condition and drastically reduces their prognosis in terms of recovery or even survival. Therefore, in clinical work one should be aware of the possibility of such a complication, know predisposing factors and not lose diagnostic alertness even when the patient's clinical condition improves.

Diagnosis of Salmonella spp. sepsis should suggest that physicians monitor patients closely, perform additional imaging tests and monitor vital signs for early detection of lifethreatening complications. 


\section{ACKNOWLEDGEMENTS}

Funding. No funding or sponsorship was received for this study or publication of this article. The Rapid Service Fee was funded by the Collegium Medicum in Bydgoszcz.

Authorship. All named authors meet the International Committee of Medical Journal Editors (ICMJE) criteria for authorship for this article, take responsibility for the integrity of the work as a whole, and have given their approval for this version to be published.

Author Contributions. All authors contributed to the study conception and design. Conceptualization: Katarzyna NapiórkowskaBaran; Formal analysis: Katarzyna Napiórkowska-Baran, Tomasz Rosada; Writing-original draft preparation: Katarzyna NapiórkowskaBaran, Tomasz Rosada, Ewa Alska, Supervision: Adam Wawrzeńczyk, Zbigniew Bartuzi; Treatment of the patient: Adam Wawrzeńczyk.

Disclosures. Katarzyna NapiórkowskaBaran, Tomasz Rosada, Ewa Alska, Adam Wawrzeńczyk and Zbigniew Bartuzi have nothing to disclose.

Compliance with Ethics Guidelines. Informed written consent was obtained from the patient for publication of this report and any accompanying images.

Data Availability. The datasets generated during and/or analysed during the current study are available from the corresponding author on reasonable request.

Open Access. This article is licensed under a Creative Commons Attribution-NonCommercial 4.0 International License, which permits any non-commercial use, sharing, adaptation, distribution and reproduction in any medium or format, as long as you give appropriate credit to the original author(s) and the source, provide a link to the Creative Commons licence, and indicate if changes were made. The images or other third party material in this article are included in the article's Creative Commons licence, unless indicated otherwise in a credit line to the material. If material is not included in the article's Creative Commons licence and your intended use is not permitted by statutory regulation or exceeds the permitted use, you will need to obtain permission directly from the copyright holder. To view a copy of this licence, visit http://creativecommons.org/licenses/by$\mathrm{nc} / 4.0 /$.

\section{REFERENCES}

1. Fleischmann C, Scherag A, Adhikari NK, et al. Assessment of global incidence and mortality of hospital-treated sepsis. Current estimates and limitations. Am J Respir Crit Care Med. 2016;193(3): 259-72.

2. Rello J, Valenzuela-Sánchez F, Ruiz-Rodriguez M, Moyano S. Sepsis: a review of advances in management. Adv Ther. 2017;34(11):2393-411.

3. Soravia-Dunand VA, Loo VG, Salit IE. Aortitis due to Salmonella: report of 10 cases and comprehensive review of the literature. Clin Infect Dis. 1999;29:862-8.

4. McKinney JS. Salmonella. In: Kliegman RM, Behrman RE, Jenson HB, Stanton B, editors. Nelson textbook of pediatrics. Philadelphia; 2007. chap. 225, pp. 1496-507.

5. Dekker JP, Frank KM. Salmonella, Shigella and Yersinia. Clin Lab Med. 2015;35(2):225-46.

6. Crump JA, Sjölund-Karlsson M, Gordon MA, Parry CM. Epidemiology, clinical presentation, laboratory diagnosis, antimicrobial resistance, and antimicrobial management of invasive salmonella infections. Clin Microbiol Rev. 2015;28(4):901-37.

7. Dunstan SJ, Hue NT, Han B, et al. Variation at HLADRB1 is associated with resistance to enteric fever. Nat Genet. 2014;46(12):1333-6.

8. Nana P, Spanos K, Dakis K, Brodis A, Kouvelos G. Imaging predictive factors of abdominal aortic aneurysm growth. J Clin Med. 2021;10(9):1917.

9. Kordzadeh A, Rhodes KM, Hanif MA, et al. Ruptured cryptogenic mycotic abdominal aortic aneurysm by Salmonella enteritidis. Ann Vasc Surg. 2013;27(7):973. 
10. Lao W-F, Huang C-H, Lin C-H, et al. Endovascular stent graft repair for a salmonella-infected aneurysm of thoracic aorta. Ann Vasc Surg. 2012;26(5): 731 .

11. Hartman V, Jiang $H$, Thomas B. Successful endovascuar repair of an abdominal mycotic aneurysm. A case report Acta Chir Belg. 2009;109: 788-90.

12. Fórmankiewicz B, Jawień A. The role of screening in early diagnosis of abdominal aortic aneurysym. Acta Angiol. 2012;18(1):1-8.

13. Lee C-H, Hsieh H-C, Ko P-J, Chou A-H, Sheng-Yueh $\mathrm{Yu}$. Treatment of infected abdominal aortic aneurysm caused by Salmonella. Ann Vasc Surg. 2014;28(1):217-26.

14. Underman AE. Salmonellosis. In: Kellerman RD, Rakel DP, editors. Conn's Current Therapy 2019. Philadelphia; 2019. pp. 612-15.

\section{Publisher's Note}

Springer Nature remains neutral with regard to jurisdictional claims in published maps and institutional affiliations. 